\title{
Sidec electron tomography: bridging the gap between light microscopy and atomic structure determinations
}

\author{
Margaretha Gadnell \& Kerstin Gunnarsson \\ Sidec electron tomography (SET) is an innovative approach to three-dimensional (3D) protein visualization. \\ SET uses low-dose radiation to produce images of individual proteins or macromolecular complexes- \\ including integral membrane proteins- in their cellular context.
}

Protein binding and conformation are vital to all processes in the cell, and there is a fundamental need to understand these events on a functional level. Most analytical techniques, however, are indirect or interfere with the object under investigation. Furthermore, traditional techniques often present averaged data from several events.

SET provides a new approach for the study of individual proteins as well as protein complexes, revealing properties such as shape, binding domains and flexibility. By taking images of individual proteins and protein complexes, conformational distribution of flexible molecules can be revealed. Most importantly, SET analysis allows the visualization of membrane proteins in their natural cellular context. SET is now the only method that can provide this information. SET can be used in a diverse array of applications and has been applied both in drug discovery and in biopharmaceutical applications (Table 1). Proteins can be analyzed in a variety of contexts: in buffer solutions, in cells or in tissue samples.

Electron tomography is an established method that uses transmission electron microscopy to take multiple two-dimensional pictures of a frozen sample. These are then assembled into 3D images by reconstruction algorithms. The unique feature of SET is that it uses a proprietary algorithm (Sidec COMET) that can reconstruct high-resolution images from low-dose electron micrographs by an iterative calculation process. An advantage of this process is that lowdose radiation used during the image capture stage is sufficient to produce images with a resolution approaching $2 \mathrm{~nm}$. SET requires only small quantities of sample material: $25 \mu \mathrm{l}$ of a $1 \mathrm{mg} / \mathrm{ml}$ solution is usually enough. Whereas X-ray crystallography, the gold standard for atomic-level protein visualization, requires milligram quantities of protein that have been processed by purification and crystallization. This precludes visualization of protein complexes in situ. SET is available through Sidec Technologies AB on a contract research basis.

\section{Principles of analysis}

A challenge with using electron microscopy approaches for structure determination is the tradeoff between obtaining high-resolu-

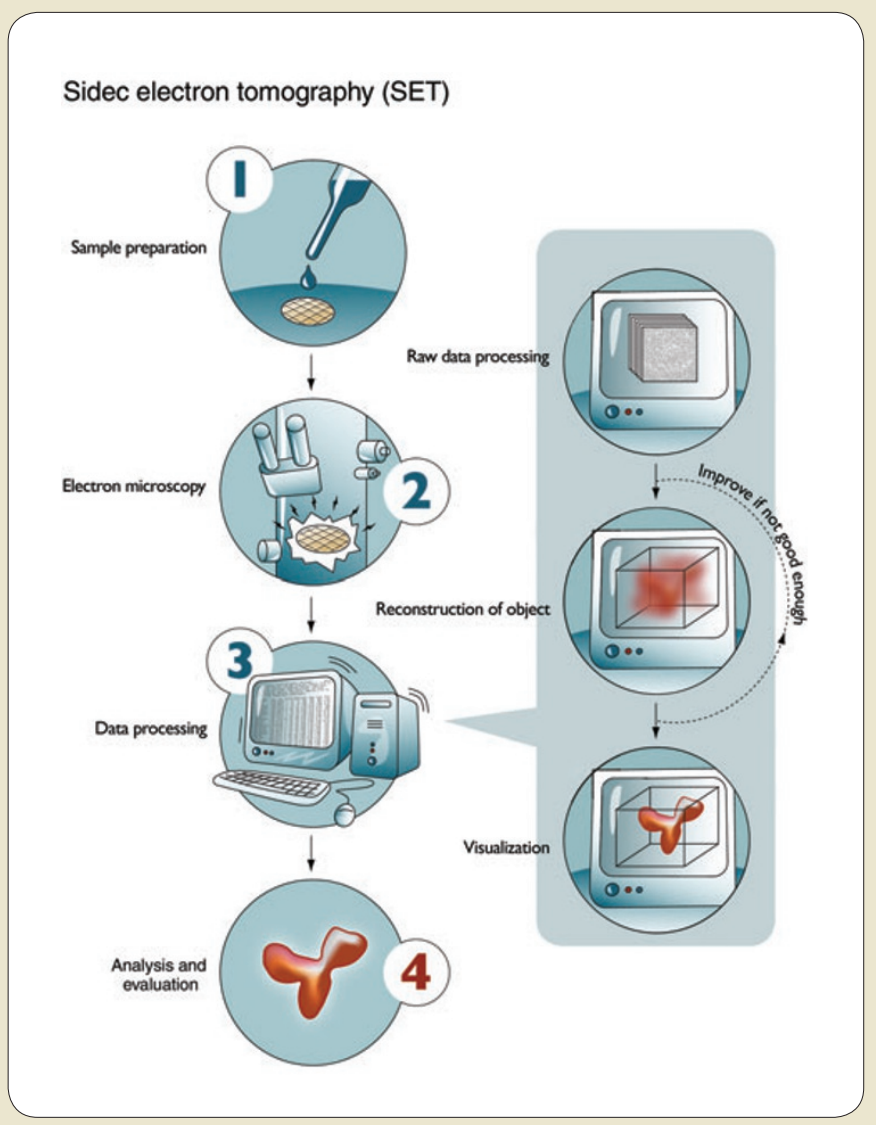

Figure 1 | SET analysis is performed in four stages. First, proteins in solution are applied as a thin film onto a sample grid and flash frozen in liquid ethane. In situ samples are initially fixed by perfusion or immersion and frozen in liquid ethane before they are cryosectioned, labeled with contrasting agent and embedded on a sample grid. Second, a tilt series of micrographs is taken at low-dose radiation using transmission electron microscopy. Third, these micrographs are aligned and assembled into $3 D$ images and refined using the Sidec COMET algorithm. This iterative process refines the images, typically to a resolution of $20-30 \AA$. Fourth, the researcher can then select molecules of interest, rotate them through $360^{\circ}$ and analyze their conformations in three dimensions. 
Table 1 | A selection of Sidec's customer projects to date

\begin{tabular}{|c|c|c|}
\hline Investigation & Results shown & Use \\
\hline $\begin{array}{l}\text { Receptor complex } \\
\text { formation }\end{array}$ & Order of assembly & $\begin{array}{l}\text { Develop } \\
\text { antagonist }\end{array}$ \\
\hline Ion-channel formation & Subunit assembly & Validate cell assay \\
\hline Glycosylation & Description of binding area & Patent \\
\hline Polymer formation & Arrangement of subunits & $\begin{array}{l}\text { Prevent polymer } \\
\text { formation }\end{array}$ \\
\hline Binding location & Binding domain & $\begin{array}{l}\text { Prevent } \\
\text { interaction }\end{array}$ \\
\hline $\begin{array}{l}\text { Shape of } \\
\text { noncrystallized protein }\end{array}$ & Conformation family & $\begin{array}{l}\text { Prevent formation } \\
\text { of active complex }\end{array}$ \\
\hline Effects of binding & $\begin{array}{l}\text { Conformation of antigen with } \\
\text { or without antibody bound }\end{array}$ & $\begin{array}{l}\text { Develop } \\
\text { therapeutic } \\
\text { antibody }\end{array}$ \\
\hline
\end{tabular}

tion images and minimizing radiation damage. SET circumvents this problem by using the proprietary constrained maximum entropy algorithm, Sidec COMET. This algorithm works as a mathematical filter on the preliminary $3 \mathrm{D}$ construction to maximize the signal-tonoise ratio of the images, thus improving the fidelity of $3 \mathrm{D}$ reconstructions and filtering much of the background noise in electron micrographs. In contrast to techniques such as 'single-particle analysis', SET reconstructs individual molecules from a sample volume instead of using averaging reconstruction models, making it possible to analyze the particular conformational changes of individual proteins as they bind and interact with one another.

\section{Sample preparation and experimental considerations}

SET analysis can be used to investigate biomolecules and their complexes in different samples. Sample preparation techniques are designed to minimize artifacts.

In situ samples require labeling with gold-conjugated antibodies to locate the protein of interest among other cell proteins. Cell cultures and tissue biopsies are fixed, cryoprotected and frozen before being sectioned with a cryoultramicrotome. Typically, a $0.5-\mathrm{mm}$ cell pellet or tissue section is used. The thin slices are incubated with primary and secondary antibodies, lightly stained and embedded in polyvinyl alcohol before tomography is performed. For in situ samples, the gold-labeled secondary antibody is located on a twodimensional electron micrograph image, which is then subjected to Sidec's unique reconstruction process to reveal the protein and its neighboring proteins in three dimensions. In addition to immunolabeling, molecular size and characteristic structural features are used to confirm the identity of a protein. In vitro samples, for example, protein buffer solutions, are flash-frozen in thin layers to maintain the proteins in their natural conformation. The rapid freezing prevents formation of crystalline ice in the sample, thus minimizing distortion of the shape of the proteins. For in vitro samples, a total of $25 \mu \mathrm{l}$ at a sample concentration of $1 \mathrm{mg} / \mathrm{ml}$ will be needed. A sample purity of $75 \%$ or more is recommended, as impurities make it more difficult and time-consuming to identify the protein of interest in the analysis phase.

\section{New insights into disease mechanisms}

SET has been applied in clinical research to understand the molecular mechanism behind glomerular capillary permeability in the kidneys, providing valuable insights into the basis of proteinuria ${ }^{1}$.

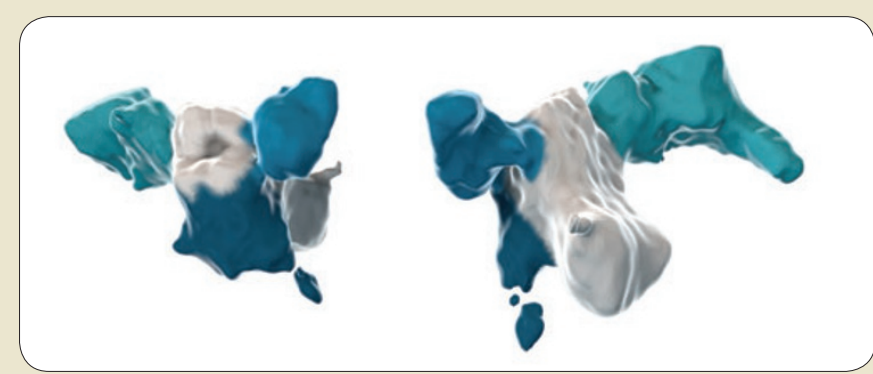

Figure 2 | I on channel visualized in situ. SET analysis resolved the size and structure of the ion channel embedded in the plasma membrane and indicated that the macromolecular complex has a tetrameric structure (white) with a central pore (gray). Extracellular components are depicted pointing upward and intracellular components downward. The lipid bilayer that extends over the neck region of the protein complex is not visualized here owing to the staining method used. Blue proteins are antibodies used for immunolabeling the ion channel.

In a collaboration between the University of Helsinki, Karolinska Institutet, University of Oulo and University of California at Davis, SET was used to image the slit diaphragm in the kidney, revealing the importance of the transmembrane protein nephrin ${ }^{1}$. Comparison between normal tissue from human, rat and mouse with tissue from patients with congenital nephritic syndrome of the Finnish type (NPHS1) and nephrin-knockout mice showed that this structure could not be formed in nephrin-deficient tissue, leading to proteinuria.

\section{Insitustudy of an ion channel complex}

With over $75 \%$ of drugs on the market today targeting membranebound proteins, there are clear benefits to technologies that can visualize these proteins in situ. SET provides a rapid method for protein conformational analysis of in situ macromolecular complexes.

In a test case with AstraZeneca, SET was used to reconstruct 3D images of ion channels in situ from cell lines and whole-tissue biopsy specimens ${ }^{2}$. Structural arrangements, such as subunit assembly, are crucial for the functioning of ion channels. SET successfully resolved the volume and structural features of the target complex, showing that it was a tetrameric channel with a central pore (Fig. 2). SET showed protein conformational states of fully associated channels and proteins in the process of forming an ion channel.

\section{Conclusions}

SET is a valuable technology for 3D visualization of individual proteins and macromolecular complexes. SET can thus further our understanding of the mechanisms of protein interactions and dynamics at the molecular level, which is a prerequisite for developing predictable model systems. This in turn will improve confidence when making decisions along the research and development process before substantial financial investments are made.

\section{ACKNOWLEDGMENTS}

SET was developed from research conducted at the Karolinska Institute in Stockholm, Sweden, and is commercially available through Sidec Molecular Imaging.

1. Wartiovaara, J. et al. Nephrin strands contribute to a porous slit diaphragm scaffold as revealed by electron tomography. J. Cin. Invest. 114, 1475-1483 (2004).

2. Banyay, M. et al. Three-dimensional imaging of in situ specimens with low-dose electron tomography to analyze protein conformation. Assay Drug Dev. Technol. 2, 561-567 (2004). 